\title{
NOCIONES Y PRÁCTICAS DE EVALUACIÓN DE LOS DOCENTES DE EDUCACIÓN FÍSICA DE LOS NIVELES DE ENSEÑANZA BÁSICA Y MEDIA EN LOS COLEGIOS PÚBLICOS DE LA LOCALIDAD DE USME
}

\author{
Juan Guillermo Bernal Gordillo \\ Estudiante de la Maestría en Ciencias y Tecnologías del Deporte y la Actividad Física, en la \\ Universidad Manuela Beltrán. Esp. Pedagogía de la Lúdica de la Fundación Universitaria Los Libertadores. \\ juanguillermob@yahoo.com.ar \\ Oscar Mauricio Rodríguez Pinzón \\ Estudiante de la Maestría en Ciencias y Tecnologías del Deporte y la Actividad Física, \\ en la Universidad Manuela Beltrán. Licenciado en Educación Física, de la Universidad CENDA. \\ oscarazul6@hotmail.com \\ Marco Vinicio Gutiérrez Casas \\ Dr. Honoris Causa del Consejo Iberoamericano
}

Recibido: 18-12-2014, aprobado: 09-02-2016, última versión: 09-02-2016¹

\section{RESUMEN}

La propuesta de investigación caracteriza las prácticas evaluativas y las concepciones que sobre evaluación poseen los docentes de Educación Física dentro de sus prácticas pedagógicas en los colegios oficiales de la localidad de Usme adscritos a la Secretaria de Educación del Distrito (S.E.D.). Contempla un diseño de investigación mixto de orden explicativo secuencial, con alcances de tipo exploratorio, descriptivo y explicativo: Una fase cuantitativa; utiliza el cuestionario como instrumento de recolección de datos. Otra, de tipo, cualitativo, emplea la entrevista formalizada. Se encontró que las concepciones y las prácticas evaluativas de los docentes de Educación Física de los colegios públicos de la localidad de Usme apuntan a una formación humanista, dando prevalencia a la dimensión axiológica, a pesar de que el docente emplee con frecuencia Test o pruebas físicas. Sin embargo, se desatiende el ámbito cognitivo del alumno, lo que puede llegar a considerar que no se promueven enfoques de aprendizaje profundos en los estudiantes de educación física.

Palabras clave: Evaluación educativa, método de evaluación, instrumentos de evaluación, educación física, prácticas evaluativas.

Este trabajo es resultado de investigación para obtener el titulo de maestría en Ciencias y Tecnologías del Deporte y la Actividad Física en la Universidad Manuela Beltrán. 


\section{ABSTRACT}

The research proposal characterizes the evaluation practices and assessment conceptions have the physical education teachers provided in their teaching in state schools in the Usme assigned to the Secretary of Education District (SED). Contemplates a mixed research design explanatory sequential order, with ranges of exploratory, descriptive and explanatory type. A quantitative phase; using the questionnaire as a tool for data collection. Another, type, quality, uses the formalized interview. It was found that the conceptions and evaluative practices of teachers of physical education in public schools of the locality of Usme it point to a formation humanist, giving prevalence to the axiological dimension, even though teachers frequently use Test or physical evidence. Likewise, given the assessment tools used, the cognitive domain of students is neglected, it may come to regard that no promote deep learning approaches in physical education students.

Keywords: Educational evaluation, method of assessment, evaluation tools, physical education, evaluative practices.

\section{INTRODUCCIÓN*}

$\mathrm{E}$ n el campo de la educación física, el proceso de evaluar, tal como lo expresan Bolívar y Camacho (1998) es un «proceso permanente a través del cual se obtiene información confiable para valorar el estado de los diferentes aspectos y componentes curriculares orientados a fortalecer los elementos positivos y corregir aquellos en los cuales existen problemas»; sin embargo, la evaluación dentro de la cotidianidad del docente de educación física no es clara ni generalizable en sus prácticas, pues puede estar orientada sobre la observación o la emisión de juicios y/o valoraciones de tipo subjetivo sobre el estudiante, sustentadas sobre una evaluación cualitativa y/o sobre la apreciación de la evaluación como sinónimo de «medición» y «calificación»; es decir, una evaluación orientada hacia lo cuantitativo basada en test y/o pruebas estandarizadas.

Es por ello que resulta necesario indagar desde la opinión del docente de educación física y desde su labor pedagógica cotidiana en con-

\section{8}

\footnotetext{
Artículo corto de investigación, adherido al macroproyecto de investigación: «Prácticas evaluativas de los docentes de educación física para detectar talentos deportivos en los colegios públicos de Bogotá», llevado a cabo en la Universidad Manuela Beltrán, durante el periodo 20132015, en la línea de profundización «Actividad Física y deporte en el ámbito escolar, comunitario y contextos no convencionales».
}

junción con las realidades de las prácticas evaluativas en educación física tanto teóricas como prácticas,

- ¿Cuáles son las nociones y enfoques de evaluación y las prácticas evaluativas que tienen los docentes de Educación Física en la educación básica y media de los colegios públicos en la localidad de Usme?

La posibilidad de poder contar con un insumo de información tan importante permite ahondar en las concepciones propias de temas como la evaluación del aprendizaje en educación física, las finalidades de la evaluación, los instrumentos, las estrategias y las técnicas de evaluación y el objeto de la evaluación, entre otros; de igual manera, sentar bases sólidas y de fuerte argumento acerca de los fines de la educación física en cuanto a los conocimientos promovidos en los estudiantes; así mismo, poder formular estrategias para el fortalecimiento de las prácticas evaluativas de los docentes de educación física en los colegios públicos de la localidad de Usme que promuevan enfoques profundos de aprendizaje en sus alumnos.

\subsection{Objetivos}

Objetivo general:

- Caracterizar las prácticas evaluativas y los enfoques de aprendizaje en las prác- 
ticas pedagógicas ejercidas por los profesores de educación física en la educación básica y media de los colegios públicos de Usme con el fin de proponer estrategias pedagógicas que fortalezcan las prácticas de evaluación que promuevan enfoques profundos de aprendizaje en los estudiantes.

Objetivos específicos:

- Identificar las nociones que sobre evaluación tienen los profesores de Educación Física en la educación básica y media de los colegios públicos de Usme.

- Determinar las estrategias de evaluación de los profesores de educación física en la educación básica y media de los colegios públicos de Usme.

- Proponer estrategias para el fortalecimiento de las prácticas evaluativas de los profesores de educación física en la educación básica y media de los colegios públicos de Usme, que promuevan enfoques profundos de aprendizaje.

\section{MÉtodo}

La investigación tiene un diseño mixto de orden explicativo secuencial que, siguiendo a Creswell y Plano-Clark (2007), tiene dos etapas, una primera fase que es cuantitativa; utiliza el cuestionario como instrumento de recolección de datos y con él su posterior análisis de datos cuantitativos. Este instrumento fue elaborado y diseñado por Oscar Fabián Rubiano Espinosa en el marco del macroproyecto de investigación «Prácticas evaluativas de los docentes de educación física para detectar talentos deportivos en los colegios públicos de Bogotá», en la Universidad Manuela Beltrán, el cual contiene una serie de preguntas cerradas y abiertas que indagan sobre la manera como los docentes llevan a cabo la evaluación en la asignatura de educación física.
La muestra son 30 docentes de educación física titulares en básica secundaria y media de las dos jornadas (mañana y tarde) de cada una de las instituciones educativas, conformada mediante muestreo probabilístico estratificado.

La segunda fase, de tipo cualitativo, se desarrollará bajo el «Modelo de selección de participantes». Se opta por la aplicación de este modelo, porque se emplean los datos cuantitativos obtenidos en la primera etapa para identificar y seleccionar intencionalmente a los docentes de educación física, recreación y deporte, como muestra de expertos. Se utilizó como instrumento de recolección de datos cualitativos la «Entrevista Formalizada». La aplicación del cuestionario y la entrevista se efectuó dentro de las fechas del mes de julio al mes de noviembre del año 2014. El análisis de los datos cuantitativos se realizó bajo el empleo de estadística descriptiva. Para el análisis de datos de la fase cualitativa, se utilizó el proceso descrito por Hernández Sampieri (2006) conocido como «Codificación de preguntas abiertas».

\section{La eVALUACión en educación física}

Para un mejor entendimiento del tema investigado, nos referiremos a tres ámbitos de estudio: Internacional, nacional y distrital.

En el ámbito internacional, uno de los momentos históricos que marcó el auge de las investigaciones en evaluación fue la Reforma Educativa surgida en Europa en el año 1993; hecho que dio paso a que distintos autores, ejemplo: Blázquez (1993), Díaz (1993), Martínez, M. (1993), Sebastiani Obrador (1993), López Pastor, V. (2000), Fraile, A. (2002), Pedraza, M., \& cols. (2002), Hidalgo, P. (2005), Martin Recio, F. (2010), Posada \& cols. (2010), perfilaran sus investigaciones sobre evaluación en educación física; es así como Díaz (1993) en su investigación de tipo descriptivo documental e histórico «La evaluación de la Educación Física en el 
tercer nivel de concreción de la reforma educativa» analizó y describió los diferentes aspectos que deben ser motivo de evaluación en dicha reforma, entre ellos: El objeto de la evaluación, las funciones de la evaluación, sus finalidades, el momento de la evaluación y los instrumentos o procedimientos empleados en la misma. Finalmente, en su estudio orienta al profesor sobre los instrumentos de evaluación y formas de proceder más aconsejables en función de los contenidos impartidos, entre ellos: Listas de control, escalas de clasificación, muestreos de tiempo, exámenes escritos, pruebas de ejecución test o baterías de test.

En el ámbito nacional, se destaca el estudio "Concepciones sobre evaluación y sus estrategias en las prácticas evaluativas en el área de educación física» realizado por Duque Escobar (2003) en la ciudad de Manizales; en esta investigación etnográfica, el investigador trató de reconstruir el sentido de las prácticas evaluativas en el área de educación física, partiendo de las concepciones que sobre el área manejan los profesores.

Se aplicaron los instrumentos (entrevistas de audio a los maestros y relatos en audio de los alumnos) para recopilar la información y luego categorizarla, con sus tendencias en particular; al analizar la información y confrontarla con la realidad, el referente teórico y los argumentos de los actores se procedió a la reconstrucción teórica y el autor logró determinar que «los docentes en general no son críticos en aspectos epistemológicos relacionados con objetivos de la evaluación como el para qué, el cómo, el cuándo, el dónde» (p. 121).

60 Así mismo, la investigación mostró que la evaluación en educación física se encuentra estrechamente relacionada con modelos tradicionales asociados a lo cuantitativo, desconociendo procesos axiológicos y formativos en el estudiante.
En el ámbito distrital, en la ciudad de Bogotá, Guio (2011) realizó el proyecto «Concepciones y prácticas evaluativas de los docentes de educación física en colegios distritales de la localidad de Usaquén», cuyo diseño investigativo de orden cualitativo y mediante un muestreo por conveniencia entrevistó a 16 docentes de las 11 instituciones públicas de la localidad de Usaquén. En esta investigación se logra hacer una descripción desde el sentir de los docentes de educación física de los colegios oficiales de esta localidad. De igual manera, describe cómo los docentes realizan las prácticas evaluativas y sus concepciones respecto a la evaluación en colegios de este sector. Dentro de las conclusiones se encontró que la tendencia de estos docentes es evaluar de forma cualitativa los procesos centrados en los valores, actitudes y comportamientos, aun cuando se siguen utilizando herramientas como test y pruebas físicas, predomina el carácter humanístico y personal en la evaluación.

En resumen, en los ámbitos internacional, nacional y distrital se encuentran referentes de investigación sobre evaluación en el contexto educativo; sin embargo, es reducido el número de estudios de investigación que indaguen sobre evaluación en educación física. De allí, la necesidad de investigar sobre las prácticas evaluativas de los docentes de educación física de los colegios públicos de la localidad de Usme y con ello propiciar la posibilidad de extrapolar los resultados obtenidos en otros contextos, que permitan la reflexión, la discusión y la construcción de conocimientos en este tema.

\section{Muestra}

De acuerdo al muestreo probabilístico estratificado, de un total de 88 docentes de educación física, recreación y deporte del nivel de enseñanza básica y media, agrupados en 35 instituciones educativas oficiales de Usme; la muestra la conforman 30 docentes, a los cuales se les 
aplicó un cuestionario; del total de encuestados se obtuvo una segunda muestra, bajo el Modelo de selección de participantes, para conformar una muestra de expertos, a quienes se les realiza una entrevista formalizada.

El cuestionario tuvo como fin: Caracterizar la manera en que se evalúa en educación física en los colegios públicos de Bogotá. Para ello, se tuvo en cuenta el conjunto de preguntas sobre: Edad, sexo, nivel educativo de los docentes de educación física, concepciones de evaluación, finalidades de la evaluación en educación física, objeto de la evaluación en educación física, experiencias significativas en las prácticas de evaluación y procedimientos de evaluación en educación física.

La entrevista formalizada tuvo como fin: Interpretar y explicar los resultados significativos, sorprendentes o «límites» encontrados en la primera etapa cuantitativa. Para ello, se tendrán en cuenta las siguientes categorías: La evaluación en el ámbito educativo, la evaluación en educación física y las prácticas evaluativas en educación física.

\section{Resultados}

Se encontró que el $60 \%$ de la población estudio se encuentra entre los 30 y 39 años de edad; así mismo, el $90 \%$ de los docentes de educación física de Usme son de género masculino. En cuanto a la experiencia como docente de educación física, un 46,7\% tiene experiencia de 11 a 15 años; por otra parte se puede señalar que el 63\% de la población tiene formación en Pregrado, es decir Licenciados o Profesionales, un 30\% son Especialistas y un 7\% tiene Maestría.

Sobre las concepciones de evaluación, los resultados obtenidos, como se describen en la Tabla 1, muestran que un $23,33 \%$ le atribuyen la mayor importancia a la evaluación como una «oportunidad de aprendizaje», sin embargo, no es un porcentaje representativo; en menor importancia que la anterior, un 30\% consideran la evaluación para «establecer criterios claros para que el estudiante los conozca y así se puedan obtener las calificaciones»; en contraposición, un $40 \%$ consideran que la evaluación no debe ser vista como un mecanismo para «clasificar a los estudiantes según los desempeños altos - medios - bajos».

Tabla 1

Concepciones sobre evaluación

\begin{tabular}{|c|c|c|c|c|c|c|c|}
\hline & $\begin{array}{c}1 \text { más } \\
\text { importante }\end{array}$ & 2 & 3 & 4 & 5 & 6 & $\begin{array}{c}7 \text { menos } \\
\text { importante }\end{array}$ \\
\hline & F.R & F.R & F.R & F.R & F.R & F.R & F.R \\
\hline $\begin{array}{l}\text { Recurso estudiante demuestre su } \\
\text { aprendizaje }\end{array}$ & $20,0 \%$ & $20,00 \%$ & $10,00 \%$ & $6,67 \%$ & $16,67 \%$ & $3,33 \%$ & $23,33 \%$ \\
\hline $\begin{array}{l}\text { Establecer criterios para calificar a } \\
\text { estudiantes }\end{array}$ & $20,00 \%$ & $20,00 \%$ & $30,00 \%$ & $13,33 \%$ & $6,67 \%$ & $6,67 \%$ & $3,33 \%$ \\
\hline Normatividad institucional & $10,00 \%$ & $6,67 \%$ & $6,67 \%$ & $13,33 \%$ & $16,67 \%$ & $23,33 \%$ & $23,33 \%$ \\
\hline $\begin{array}{l}\text { Participación del estudiante en } \\
\text { evaluación }\end{array}$ & $6,67 \%$ & $6,67 \%$ & $23,33 \%$ & $20,00 \%$ & $13,33 \%$ & $20,00 \%$ & $10,00 \%$ \\
\hline Diagnóstico conocimientos previos & $16,67 \%$ & $20,00 \%$ & $13,33 \%$ & $16,67 \%$ & $23,33 \%$ & $6,67 \%$ & $3,33 \%$ \\
\hline $\begin{array}{l}\text { Clasificar estudiantes según } \\
\text { desempeños }\end{array}$ & $3,33 \%$ & $13,33 \%$ & $0,00 \%$ & $6,67 \%$ & $6,67 \%$ & $30,00 \%$ & $40,00 \%$ \\
\hline Oportunidad de aprendizaje & $23,33 \%$ & $13,33 \%$ & $10,00 \%$ & $20,00 \%$ & $10,00 \%$ & $10,00 \%$ & $13,33 \%$ \\
\hline $\begin{array}{l}\text { * Frecuencia Relativa (FR): “Las frec } \\
\text { Sampieri, 2006). }\end{array}$ & ias relativ & son los & rrcenta & de cas & en cada & categoría & (Hernández \\
\hline
\end{tabular}

Fuente: Los autores. 
Con respecto a las finalidades de la evaluación, se encontró que un $46,67 \%$ considera muy importante la evaluación para "verificar qué tanto ha aprendido el estudiante»; un 26,67\% para «valorar qué tanto ha servido su sistema de enseñanza» y un 30\% de los encuestados señalan que la evaluación debe servir para «motivar e incentivar al estudiante».
Por otra parte un $43,33 \%$ de los docentes consideran que la evaluación no es importante para «obtener datos para investigar»; así mismo, un $46,67 \%$ expresaron que la evaluación no es tan importante para «asignar calificaciones» (Véase la Tabla 2).

Tabla 2

Finalidades de la evaluación

\begin{tabular}{|l|c|c|c|c|c|c|c|c|}
\hline & $\begin{array}{c}\mathbf{1} \text { más } \\
\text { importante }\end{array}$ & $\mathbf{2}$ & $\mathbf{3}$ & $\mathbf{4}$ & $\mathbf{5}$ & $\mathbf{6}$ & $\mathbf{7}$ & $\begin{array}{c}\mathbf{8} \text { menos } \\
\text { importante }\end{array}$ \\
\cline { 2 - 9 } & $\mathbf{F . R}$ & $\mathbf{F . R}$ & $\mathbf{F . R}$ & $\mathbf{F . R}$ & $\mathbf{F . R}$ & $\mathbf{F . R}$ & F.R & F.R \\
\hline Verificar aprendizajes & $46,67 \%$ & $20,00 \%$ & $3,33 \%$ & $20,00 \%$ & $6,67 \%$ & $3,33 \%$ & $0,00 \%$ & $0,00 \%$ \\
\hline $\begin{array}{l}\text { Determinar rendimiento } \\
\text { frente a parámetros }\end{array}$ & $20,00 \%$ & $20,00 \%$ & $13,33 \%$ & $20,00 \%$ & $6,67 \%$ & $20,00 \%$ & $0,00 \%$ & $0,00 \%$ \\
\hline Valorar sistema de enseñanza & $3,33 \%$ & $26,67 \%$ & $16,67 \%$ & $23,33 \%$ & $16,67 \%$ & $3,33 \%$ & $6,67 \%$ & $3,33 \%$ \\
\hline Detectar talentos deportivos & $0,00 \%$ & $6,67 \%$ & $6,67 \%$ & $6,67 \%$ & $33,33 \%$ & $20,00 \%$ & $10,00 \%$ & $16,67 \%$ \\
\hline Motivar al estudiante & $20,00 \%$ & $16,67 \%$ & $30,00 \%$ & $6,67 \%$ & $10,00 \%$ & $6,67 \%$ & $6,67 \%$ & $3,33 \%$ \\
\hline Clasificar estudiante & $3,33 \%$ & $3,33 \%$ & $16,67 \%$ & $13,33 \%$ & $10,00 \%$ & $20,00 \%$ & $23,33 \%$ & $10,00 \%$ \\
\hline Asignar calificaciones & $0,00 \%$ & $3,33 \%$ & $6,67 \%$ & $3,33 \%$ & $10,00 \%$ & $20,00 \%$ & $10,00 \%$ & $46,67 \%$ \\
\hline Investigación docente & $6,67 \%$ & $3,33 \%$ & $10,00 \%$ & $6,67 \%$ & $6,67 \%$ & $6,67 \%$ & $43,33 \%$ & $16,67 \%$ \\
\hline
\end{tabular}

Fuente: Los autores.

Respecto a los aspectos que se evalúan en Educación Física, como se observa en la Tabla 3, es muy frecuente que el $76,67 \%$ de los docentes de educación física evalúen «valores (responsabilidad, solidaridad)»; un 63,33\% evalúa con mucha frecuencia en sus estudiantes la «res- ponsabilidad»; los principales aspectos que no se evalúan son los relacionados con la «táctica deportiva» y los que se presentan menor frecuencia de ser evaluados son los relacionados con «anatomía - fisiología» y «estrategia deportiva».

Tabla 3

Aspectos a evaluar en Educación Física

\begin{tabular}{|l|c|c|c|c|}
\hline & No lo evalúo & Poco frecuente & Frecuente & Muy frecuente \\
\cline { 2 - 4 } & F.R & F.R & F.R & F.R \\
\hline Estrategia deportiva & $16,67 \%$ & $46,67 \%$ & $33,33 \%$ & $3,33 \%$ \\
\hline Cumplimiento normas de clase & $0,00 \%$ & $3,33 \%$ & $53,33 \%$ & $43,33 \%$ \\
\hline Composición corporal & $16,67 \%$ & $53,33 \%$ & $23,33 \%$ & $6,67 \%$ \\
\hline Actitudes frente a compañeros & $23,33 \%$ & $43,33 \%$ & $26,67 \%$ & $6,67 \%$ \\
\hline Cualidades físicas & $3,33 \%$ & $0,00 \%$ & $40,00 \%$ & $56,67 \%$ \\
\hline Anatomía - fisiología & $0,00 \%$ & $6,67 \%$ & $43,33 \%$ & $50,00 \%$ \\
\hline Valores & $6,67 \%$ & $56,67 \%$ & $23,33 \%$ & $13,33 \%$ \\
\hline Habilidades motrices & $0,00 \%$ & $0,00 \%$ & $23,33 \%$ & $76,67 \%$ \\
\hline
\end{tabular}




\begin{tabular}{|l|c|c|c|c|}
\hline & No lo evalúo & Poco frecuente & Frecuente & Muy frecuente \\
\cline { 2 - 5 } & F.R & F.R & F.R & F.R \\
\hline Conocimientos de deportes & $13,33 \%$ & $16,67 \%$ & $56,67 \%$ & $13,33 \%$ \\
\hline Capacidad de liderazgo & $0,00 \%$ & $16,67 \%$ & $53,33 \%$ & $30,00 \%$ \\
\hline Habilidades técnicas & $3,33 \%$ & $23,33 \%$ & $36,67 \%$ & $36,67 \%$ \\
\hline $\begin{array}{l}\text { Conocimientos sobre hábitos de } \\
\text { vida saludable }\end{array}$ & $3,33 \%$ & $20,00 \%$ & $50,00 \%$ & $26,67 \%$ \\
\hline Trabajo en equipo & $0,00 \%$ & $6,67 \%$ & $30,00 \%$ & $63,33 \%$ \\
\hline Expresión corporal & $10,00 \%$ & $23,33 \%$ & $46,67 \%$ & $20,00 \%$ \\
\hline
\end{tabular}

Fuente: Los autores.

Los procedimientos de evaluación en educación física que utilizan con mayor frecuencia son la «autoevaluación del estudiante», los «test técnico-tácticos» y la «participación de los estudiantes en eventos deportivos»; un 43,33\% no utilizan las "pruebas escritas de apareamiento» como procedimiento de evaluación; así mismo, un $40,00 \%$ no emplea las «pruebas escritas de Verdadero/falso» para evaluar a sus estudiantes, como se observa en la Tabla 4.

Tabla 4

Procedimientos de evaluación en Educación Física

\begin{tabular}{|c|c|c|c|c|}
\hline & No lo evalúo & Poco frecuente & Frecuente & Muy frecuente \\
\hline & F.R & F.R & F.R & F.R \\
\hline Listas de verificación & $10,00 \%$ & $50,00 \%$ & $40,00 \%$ & $0,00 \%$ \\
\hline Escalas de clasificación & $13,33 \%$ & $43,33 \%$ & $43,33 \%$ & $0,00 \%$ \\
\hline Técnicas de observación & $0,00 \%$ & $6,67 \%$ & $70,00 \%$ & $23,33 \%$ \\
\hline Técnicas de medición cronometraje & $13,33 \%$ & $33,33 \%$ & $40,00 \%$ & $13,33 \%$ \\
\hline Comprobación de comportamientos & $6,67 \%$ & $26,67 \%$ & $53,33 \%$ & $13,33 \%$ \\
\hline Exámenes escritos & $20,00 \%$ & $53,33 \%$ & $16,67 \%$ & $10,00 \%$ \\
\hline Pruebas V/F & $40,00 \%$ & $46,67 \%$ & $13,33 \%$ & $0,00 \%$ \\
\hline Pruebas selección múltiple & $13,33 \%$ & $63,33 \%$ & $16,67 \%$ & $6,67 \%$ \\
\hline Pruebas apareamiento & $43,33 \%$ & $46,67 \% \%$ & $10,00 \%$ & $0,00 \%$ \\
\hline Pruebas de identificación & $13,33 \%$ & $53,33 \%$ & $20,00 \%$ & $13,33 \%$ \\
\hline Pruebas preguntas abiertas & $20,00 \%$ & $46,67 \%$ & $30,00 \%$ & $3,33 \%$ \\
\hline Exámenes orales & $20,00 \%$ & $36,67 \%$ & $23,33 \%$ & $20,00 \%$ \\
\hline Evaluación grupal & $13,33 \%$ & $13,33 \%$ & $50,00 \%$ & $23,33 \%$ \\
\hline Test técnico-tácticos & $3,33 \%$ & $6,67 \%$ & $56,67 \%$ & $33,33 \%$ \\
\hline Grupos de discusión & $36,67 \%$ & $50,00 \%$ & $13,33 \%$ & $0,00 \%$ \\
\hline Participación en eventos deportivos & $6,67 \%$ & $6,67 \%$ & $53,33 \%$ & $33,33 \%$ \\
\hline Exposiciones & $16,67 \%$ & $43,33 \%$ & $33,33 \%$ & $6,67 \%$ \\
\hline $\begin{array}{l}\text { Cumplimiento responsabilidades } \\
\text { académicas }\end{array}$ & $3,33 \%$ & $20,00 \%$ & $53,33 \%$ & $23,33 \%$ \\
\hline Co-evaluación & $3,33 \%$ & $26,67 \%$ & $40,00 \%$ & $30,00 \%$ \\
\hline Autoevaluación & $0,00 \%$ & $20,00 \%$ & $40,00 \%$ & $40,00 \%$ \\
\hline
\end{tabular}

Fuente: Los autores. 


\section{Discusión}

En cuanto a las concepciones sobre evaluación los docentes de educación física de Usme, dentro de sus prácticas pedagógicas, consideran la evaluación como una oportunidad de aprendizaje para el estudiante, por lo tanto, se puede decir que manejan una evaluación de tipo Formativa o Procesual, que de acuerdo a Blázquez (1993) «es usada para motivar a los estudiantes, conocer las progresiones de sus habilidades y capacidades y diagnosticar anomalías en el desarrollo de la condición física o capacidades coordinativas»; ello da a entender que los docentes tienen en cuenta para la emisión de sus juicios los progresos realizados por los alumnos, más allá de los resultados o productos finales.

Habría que decir también que los resultados obtenidos sugieren que dentro del colectivo de docentes de educación física de Usme, la evaluación es vista como un recurso para mejorar los procesos de aprendizaje en el estudiante, mientras que la clasificación de los estudiantes es lo menos importante para los docentes al momento de conceptualizar la evaluación.

En relación con las finalidades de la evaluación se pudo evidenciar que los docentes le atribuyen a la evaluación la función de verificar qué tanto ha aprendido el estudiante, pero también la emplean como una herramienta que le permite al docente un proceso de reflexión sobre su labor docente, especialmente sobre su sistema de enseñanza, hecho que va muy de la mano con los hallazgos encontrados frente a la indagación sobre las concepciones de evaluación.

Con respecto a los aspectos que se evalúan con mayor frecuencia en educación física de los colegios públicos de Usme, puede decirse 64 tivas en: Trabajo en equipo, actitudes frente a los compañeros, cumplimiento de las reglas y normas establecidas en el curso, así como también evalúan habilidades motrices y cualidades la medida en que se encuentran similitudes en los resultados presentados por Posada, Martínez \& Ruíz (2010) quienes lograron determinar que los docentes de educación física de Manizales destacan principalmente los siguientes aspectos a evaluar: «Comportamiento, Trabajo en equipo, Capacidades condicionales o coordinativas, Asistencia del estudiante, Trabajos teórico - prácticos, Participación y Rendimiento físico».

Finalmente, al hacer mención sobre los procedimientos de evaluación que suelen utilizar los docentes de educación física de Usme, los hallazgos encontrados permitieron evidenciar que la Autoevaluación como instrumento evaluativo es uno de los más usados; de igual manera los Festivales Escolares y los MiniDeportes se convierten en una herramienta de evaluación de la participación de los estudiantes; otro instrumento de evaluación de recurrencia en las practicas evaluativas de los docentes lo constituyen los test o pruebas de ejecución de habilidades técnicas o tácticas; procedimientos que están estrechamente relacionados con los aspectos que se evalúan en estos colegios. Los procedimientos que no se emplean son las pruebas escritas de verdadero/falso y las pruebas escritas de apareamiento; por lo que se puede considerar que dentro de las prácticas evaluativas de los docentes de educación física de Usme, el ámbito cognitivo del estudiante no es objeto de evaluación. Por ello, es pertinente señalar a Chivite (1990) quien indica que el docente de educación física debe tener presente en la evaluación los tres ámbitos del estudiante y con ellos los siguientes instrumentos de evaluación: Ámbito cognitivo (exámenes teóricos, cuaderno de clase, trabajos); ámbito afectivo (autoevaluación, análisis del comportamiento); ámbito psicomotor (test cuantitativos, planillas de control). físicas; hallazgo que resulta ser interesante en
Dados los hallazgos encontrados, resulta pertinente la continuidad de la investigación en una segunda fase, de tipo cualitativo, que permita, 
desde el sentir y las opiniones de los docentes, profundizar sobre las concepciones que sobre evaluación tienen los docentes de educación física de Usme así como sus prácticas evaluativas.

\section{Conclusiones}

Los hallazgos encontrados en ésta primera fase de la investigación dan a conocer que las concepciones y las prácticas evaluativas de los docentes de Educación Física de los colegios públicos de la localidad de Usme apuntan a una formación humanista, dando prevalencia a la dimensión axiológica, a pesar de que el docente emplee con frecuencia test o pruebas físicas; lo que da cuenta de unas prácticas evaluativas orientadas hacia los ámbitos afectivo y psicomotor del alumno; sin embargo, resulta inquietante que se desatiende el ámbito cognitivo del alumno, lo que puede llegar a considerar que no se promueven enfoques de aprendizaje profundos en los estudiantes de educación física de los colegios públicos de Usme.

\section{REFERENCIAS}

Blázquez, D. (1993). Perspectivas de la evaluación en educación física y deporte. Apunts: Educación Física y Deportes. Obtenido de: http:/ / dialnet.unirioja.es/servlet/articulo?codigo=321627\& orden $=324333 \&$ info $=$ link

Díaz, J. (1993). La evaluación de la educación física en el tercer nivel de concreción de la reforma educativa. Obtenido de Educación Física y Deportes: http:/ / dialnet.unirioja.es/servlet/artic ulo? codigo $=321652 \&$ orden $=324335 \&$ info $=$ link

Fraile, A. (2002). La valoración del aprendizaje del alumnado en educación física. Obtenido de Revista Aula: http://www.uruguayeduca.edu.uy/Userfiles/P0001/File/ValorApzjeEF.pdf

Hernández Sampieri, R. (2006). Metodología de la investigación. México: Mc Graw Hill.

Duque, E. (2003). Concepciones sobre evaluación y sus estrategias en las prácticas evaluativas en el área de educación física. Centro Internacional de Educación y Desarrollo Humano (CINDE). Universidad de Manizales. Manizales.

Guio Gutiérrez, F. (2011). Concepciones y prácticas evaluativas de los docentes de educación física en colegios distritales de la localidad de Usaquén. Obtenido de http:/ / intellectum.unisabana. edu.co:8080/jspui/bitstream/10818/1660/1/Fernando_Gu_o_Guti_rrez.pdf

Hidalgo, P. (2005). La evaluación teórica en educación física: Un medio más para el aprendizaje. Obtenido de Aula de Innovación Educativa: http://europa.sim.ucm.es/compludoc/ AA?articuloId $=357164$

Posada, W., Martínez. J., \& Ruiz, L. (2010). La evaluación de la educación física y las políticas educativas en Colombia. Recuperado el 10 de agosto de 2013, de http://www.rieoei.org/ deloslectores/3777Posada.pdf 